\title{
Neoplasia de Células Dendríticas Plasmocitoides blásticas: reporte del primer caso en Ecuador
}

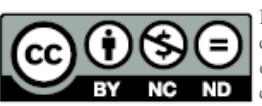

Este articulo está bajo una cencia de Creative Commons de tipo Reconocimiento - No OPEN ACCESS das 4.0 International

1.Postgrado Medicina Interna. Pontificia Universidad Católica del Ecuador.

2.Pontificia Universidad Católica del Ecuador. Hospital General Enrique Garcés, departamento de Medicina Interna

3.Pontificia Universidad Católica del Ecuador. Hospital Sociedad de Lucha contra el Cáncer (SOLCA), departamento de Hematología.

ORCID ID:

Elisa Jácome Sánchez

https:/ / orcid.org/0000-0002-3185-9112

Nelson Cevallos Salas

https:/ / orcid.org/0000-0001-8386-9243

Patricio Hidalgo Dillon

https:/ / orcid.org/0000-0003-2927-3283

Recibido: 01 - julio - 2019

Aceptado: 23 - octubre - 2019.

Publicado: 01 - diciembre - 2019

Correspondencia: Elisa Jácome Sánchez.

E-mail: elisajacome@yahoo.es

Contribución de autores: Los autores decla ran haber contribuido de manera similar en la redacción del manuscrito.

Financiamiento: Reporte financiado con fondos propios de los autores.

Conflictos de interés: Los autores declaran no poseer conflicto de interés.

\section{Resumen}

La Neoplasia de Células Dendríticas Plasmocitoides blásticas (Blastic Plasmacytoid dendritic cell neoplasm - BPDCN) es una neoplasia hematológica rara, agresiva, de difícil diagnóstico y con alta mortalidad.

Se describe el primer caso en el Ecuador de un paciente joven sin antecedentes patológicos relevantes, ingresado al servicio de Medicina Interna del Hospital Enrique Garcés por presentar máculas cutáneas, artralgias y mialgias, que se complica con derrame pleural tipo exudativo y mala mecánica respiratoria. Exámenes de extensión revelaron: Leucemia mieloide aguda de tipo $M 2$, motivo por el cual fue referido a centro oncológico de referencia para completar estudio y manejo.

Estudios citogenéticos y fenotípicos corroboraron el diagnóstico de BPDCN, se instauró tratamiento con protocolo Hyper-CVAD, sin embargo, el paciente presentó compromiso respiratorio, renal y hematológico que progresó a choque refractario y óbito.

La naturaleza agresiva de esta rara leucemia es una limitante en el tiempo para instaurar un tratamiento dirigido, determinando en la mayoría de los casos una alta mortalidad.

Palabras clave: Síndrome de Bean, Albinismo, Sangrado Gastrointestinal, Anemia,

Síndrome de HermLeucemia, célula dendrítica, linfom

\section{Abstract}

\section{Blastic Plasmacytoid Dendritic Cell Neoplasm :} First Case Report in Ecuador

Blastic Plasmacytoid Dendritic Cell Neoplasm (BPDCN) is a rare, aggressive, difficult-to-diagnose haematological neoplasm with high mortality.

The first case in Ecuador of a young patient with no relevant pathological history, admitted to the internal medicine service of the Enrique Garcés Hospital for presenting cutaneous macula, arthralgias and myalgias, which is complicated with pleural effusion type exudative and poor respiratory mechanics. Extension tests revealed: Acute myeloid leukemia type $\mathrm{M} 2$, which is why he was referred to a reference cancer center to complete study and management.

Cytogenetic and phenotypic studies corroborated the diagnosis of BPDCN, Treatment with Hyper-CVAD protocol was instituted, however, the patient presented respiratory, renal and haematological commitment that progresses to refractory and death shock.

The aggressive nature of this rare leukemia is a limitation in time to institute targeted treatment, most often determining high mortality.

Keywords: : Leukemia, dendritic cell, lymphoma 


\section{Introducción}

La Neoplasia de Células Dendríticas Plasmocitoides Blásticas (Blastic Plasmacytoid Dendritic Cell Neoplasm - BPDCN) es una rara y agresiva neoplasia hematológica de tipo CD4+/ CD56+, derivada de células dendríticas precursoras de tipo II, con curso fatal. ${ }^{(1,2)}$

Esta patología ha sido conocida con varios nombres como leucemia/linfoma de células Natural Killer (NK) blásticas, leucemia de células NK CD4+ agranular, neoplasia hematodérmica y agranular CD4+/CD56+; en el 2003 se confirma el origen celular: células dendríticas plasmacitoides tipo II. (2-7)

Son pocos los estudios epidemiológicos que describen su incidencia, representa el $0,44 \%$ de las neoplasias malignas hematológicas, el $0.7 \%$ de los linfomas cutáneos y el $6.3 \%$ de la línea celular NK. ${ }^{(8,9)}$

El primer caso reportado fue en 1994, desde entonces, 285 casos han sido documentados en la literatura médica: 120 de origen francés, 100 ingleses, 45 entre norteamericanos y canadienses, 6 holandeses, 5 españoles, 6 chinos y 3 mexicanos. ${ }^{(1-3,10-12)}$ Puede estar asociada a otras neoplasias como el linfoma no Hodgkin y la leucemia mieloide aguda, sin embargo, su verdadera incidencia es desconocida. $(1,3-5,8,9,13,14)$ A continuación presentamos el primer caso reportado en Ecuador.

\section{Presentación del Caso}

Paciente masculino de 33 años, sin antecedentes patológicos personales. Presentó un cuadro de 15 días de evolución, caracterizado por edema maleolar bilateral, máculas violáceas cutáneas de diferente localización y tamaño (Figura 1) artralgias y mialgias, motivo por el cual fue admitido en el Hospital Enrique Garcés. Durante la hospitalización hubo compromiso de la mecánica respiratoria debido a la presencia de derrame pleural. (Figura 2) Se realizó toracocentesis diagnóstica y evacuatoria, obteniéndose líquido exudativo 1500cc del lado derecho y $450 \mathrm{cc}$ del lado izquierdo. La analítica evidenció repunte leucocitario de predominio mononuclear con proteína $C$ reactiva (PCR) y procalcitonina (PCT) negativas. La biopsia cutánea reportó una vasculitis leucocitoclástica. el aspirado de medula ósea y el frotis de sangre periférica demostraron hallazgos compatibles con leucemia mieloide aguda M2. (Figura 3)

Con este diagnóstico se lo refirió al Hospital Sociedad de Lucha contra el Cáncer (SOLCA) - Quito, donde por compromiso de la función respiratoria secundaria al derrame pleural bilateral masivo (Figura 4), se lo ingresó a la Unidad de Cuidados Intensivos (UCI) para ventilación mecánica no invasiva y colocación de tubos torácicos a cada lado, se obtuvo $2550 \mathrm{ml}$ de líquido en el lado derecho y $2620 \mathrm{ml}$ en el lado izquierdo, compatibles con exudados de origen tumoral (tabla 1).

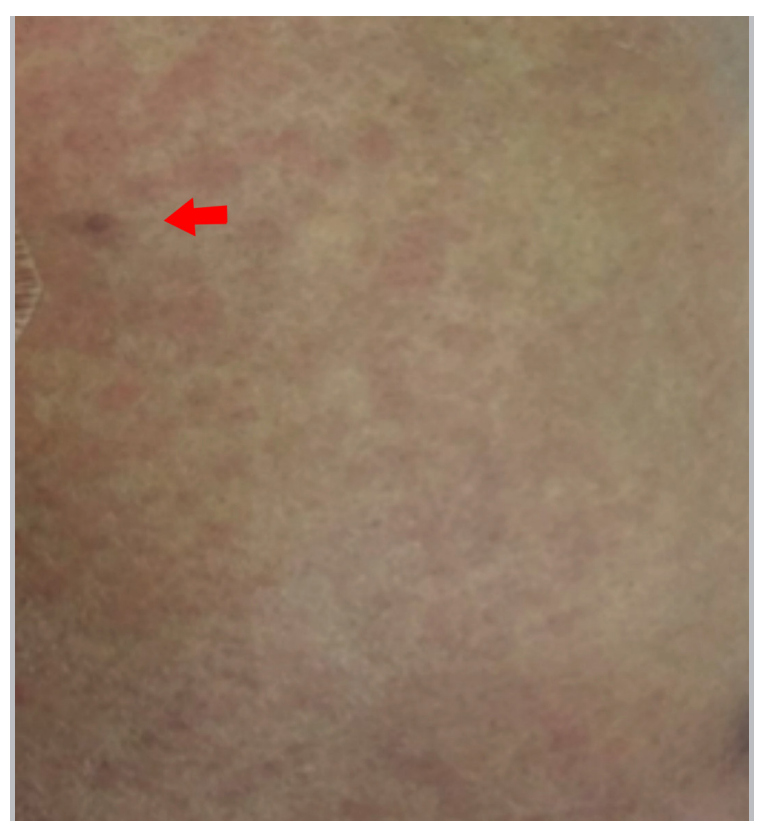

Figura 1. Lesiones dérmicas maculopapulares

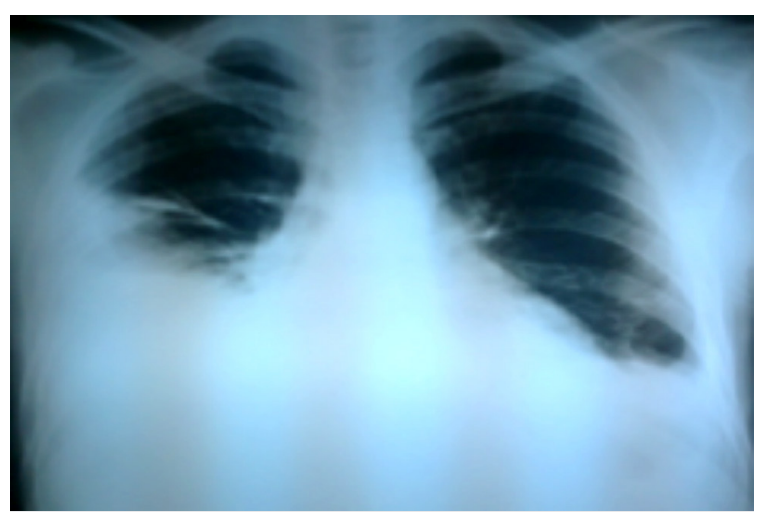

Figura 2. Derrame pleural bilateral

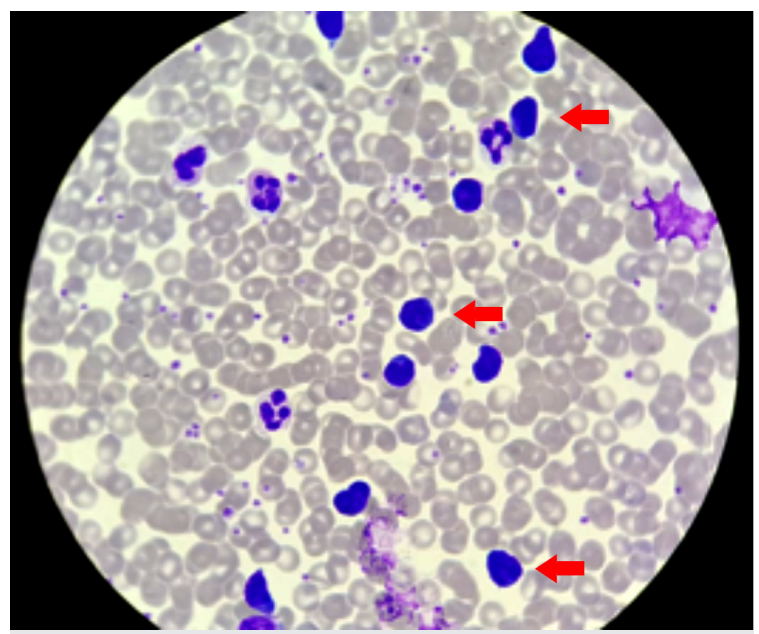

Figura 3. Aspirado de Médula ósea: se aprecia importante hipercelularidad, las fechas rojas indican a las células plasmocitoide dendrítica. 


\section{Tabla 1 - Ecocardiograma y estudios citológicos y bioquímicos de líquido pericárdico y pleural, obtenidos} durante la internación en la Unidad de terapia intensiva.

\section{ECOCARDIOGRAMA}

PSAP $42 \mathrm{~mm} / \mathrm{hg}$, valva anterior de mitral de borde libre elongada. Ventrículo izquierdo hiperdinámico, función sistólica conservada, FEVY 61\%. Función diastólica alterada en la relajación I/IV, derrame pleural con compresión mecánica a toda el área cardíaca, pericardio con ecogenicidad aumentada, derrame leve hacia región apical y posterior, separación entre las dos membranas de 13mm y de las paredes de $5 \mathrm{~mm}$.

\section{LÍQUIDO PERICÁRDICO}

Amarillo turbio

Células: $23630 \mathrm{cel} / \mathrm{mm} 3$

Mononucleares: 100\%

Glucosa: 127mg/dl

Lactato deshidrogenasa: 1169

Proteínas: $2.78 \mathrm{mg} / \mathrm{dl}$

Albúmina: $1.88 \mathrm{mg} / \mathrm{dl}$

Cultivo: Negativo

\author{
LÍQUIDO PLEURAL \\ Amarillo hemático \\ Turbio \\ Leucocitos: $18432 \mathrm{cel}(\mathrm{mm} 3$ \\ Polimorfonucleares: $10 \%$ \\ Mononucleares: $90 \%$ \\ Hematíes: $7.6 \mathrm{cel} / \mathrm{mm} 3$ \\ Hematíes normales: $99 \%$ \\ Hematíes crenados: $1 \%$ \\ ADA: $44.4 \mathrm{u} / \mathrm{l}$ \\ Lactatodeshidrogenasa: 821 \\ Glucosa: 93mg/dl \\ Proteínas: $2990 \mathrm{mg} / \mathrm{dl}$ \\ Albúmina: 1920mg/dl \\ Cultivo: Negativo
}

Fuente: Elaborado por Md Carolina Jácome

Tabla 2 - Estudios citológicos, citogenéticos y fenotípicos de la médula ósea

\begin{tabular}{|c|c|c|c|}
\hline \multicolumn{2}{|c|}{ ASPIRADO DE MÉDULA ÓSEA } & CITOMETRÍA DE FLUJO & CARIOTIPO \\
\hline \multirow[t]{7}{*}{ Serie } & Granulocítica $21 \%$ & CD56+ & $46 X Y$ \\
\hline & Linfoide $2 \%$ & CD4+ & FISH: T(9:22)(Q34,Q1 1): Negativo \\
\hline & Mieloeritroide 3:1 & CD45 + & BCR/ABL: Negativo \\
\hline & Roja 0\% & CD38 +A- & \\
\hline & Blastos $77 \%$ & CD71 DÉBIL + & \\
\hline & Celularidad monomorfa ++ & Marcadores T y B: - & \\
\hline & Población dendrítica $70.59 \%$ & $\begin{array}{c}\text { Marcadores de inmadurez: - } \\
\text { Marcadores mieloides: - }\end{array}$ & \\
\hline
\end{tabular}

En la $\mathrm{UCl}$, el paciente presentó deterioro hemodinámico súbito con taquicardia multifactorial e hipotensión arterial requiriendo vasoactivos para mantener la macrodinamia. Los resultados de las muestras obtenidas para estudio y estadiaje de la leucemia se describen en la (tabla 2).

Los resultados citogenéticos y fenotípicos confirmaron el diagnóstico de BPDCN y se inició protocolo HyperCVAD (Ciclofosfamida, Vincristina, Adriamicina y Dexametasona/ Metotrexato, Citarabina). A las 96 horas de su llegada mostró choque refractario a doble vasoactivo, con un ecocardiograma que demostró la presencia de derrame pericárdico con colapso ventricular derecho (Tabla 1).

Se realizaron 3 pericardiocentesis, la primera evacuando $300 \mathrm{ml}$ de contenido liquido turbio hemorrágico, la segunda y tercera con un volumen total de $600 \mathrm{ml}$ de las mismas características, mejorando parcialmente la macrodinamia del paciente sin embargo persistía con hiperlactatemia, acidosis metabólica, hipercalemia, falla renal severa y anuria que no respondió a diuréticos (Tabla 3). El paciente progreso a choque refractario y óbito.

\section{Discusión}

La BPDCN es una entidad rara, caracterizada por la proliferación maligna de una célula dendrítica plasmocitoide blástica. Se desconoce la real incidencia de esta patología por lo infrecuente que es, y por lo difícil de su diagnóstico. ${ }^{(8)}$

La proporción hombre/mujer es 2:2, sin embargo, Avilés $A$, et $a_{\text {., }}{ }^{(1)}$ reporta una preponderancia por el sexo masculino, siendo más común en adultos mayores con una media de edad de 67.2 años (8103 años). ${ }^{(4,8,9)}$

Literatura existente describe una diseminación cutánea y leucémica con o sin afectación de la médula ósea. Es una patología de mal pronóstico, con un curso extremadamente rápido y una sobrevida de 12 a 14 meses en el mejor de los casos, $(4,6,8,9,14,15)$ tal como nuestro paciente, el cual tuvo lesiones dérmicas con afectación medular y fallecimiento a los 30 días de instaurado el cuadro. 
Tabla3 - Hemograma y Química sanguínea al ingreso y óbito del paciente

\begin{tabular}{|lccc|}
\hline \multicolumn{2}{|c}{ Parámetro } & Ingreso & Óbito \\
Leucocitos & $(\mathrm{K} / \mathrm{UL})$ & 136290 & 145700 \\
Neutrófilos & $(\mathrm{K} / \mathrm{UL})$ & 27666 & 28702 \\
Linfocitos & $(\mathrm{K} / \mathrm{UL})$ & 36798 & 44292 \\
Monocitos & $(\mathrm{K} / \mathrm{uL})$ & 67327 & 68333 \\
Hemoglobina & $(\mathrm{g} / \mathrm{dL})$ & 16.9 & 18.5 \\
Hematocrito & $(\%)$ & 49.4 & 52.7 \\
Plaquetas & $(\mathrm{K} / \mathrm{uL})$ & 100000 & 589000 \\
TP & $(\mathrm{seg})$ & 11.3 & \\
TTP & $(\mathrm{seg})$ & 22 & \\
& & 0.94 & \\
Ácido úrico & $(\mathrm{mg} \%)$ & 7 & 4.2 \\
LDH & $(\mathrm{U} / \mathrm{L})$ & 945 & \\
Fósforo & $(\mathrm{mg} \%)$ & 5.72 & 8.48 \\
Magnesio & $(\mathrm{meq} / \mathrm{l})$ & 0.84 & 1.14 \\
Potasio & $(\mathrm{meq} / \mathrm{l})$ & & 6.34 \\
Sodio & $(\mathrm{meq} / \mathrm{l})$ & & 120 \\
Calcio total & $(\mathrm{meq} / \mathrm{l})$ & & 7.37 \\
Proteínas totales: & $(\mathrm{gr} \%)$ & 4.5 & \\
Albúmina & $(\mathrm{gr} \%)$ & 2.5 & \\
Fosfatasa alcalina & $(\mathrm{u} / \mathrm{L})$ & 492 & \\
Creatinina & $(\mathrm{mg} / \mathrm{dL})$ & 1.56 & 2.76 \\
\hline
\end{tabular}

Fuente: Elaborado por Md Carolina Jácome

La forma de presentación clínica de nuestro paciente es muy similar a uno de los casos reportados por Avilés A, et al., (1) e incluso guardan semejanzas en el diagnóstico, ya que en ambos era muy evidente la leucocitosis importante. En la mayoría de los casos, es evidente la afectación de la médula ósea y de sangre periférica junto a lesiones cutáneas, $(2,16)$ sin embargo en la analítica básica suelen cursar con pancitopenia 0 trombocitopenia ${ }^{(8)}$ lo que difiere con 10 encontrado en este caso.

Las lesiones cutáneas diseminadas se presentan apenas en el $15 \%$ de los casos, pero son las más representativas de la enfermedad y se caracterizan por asociación de nódulos, pápulas y máculas purpúricas generalizadas ${ }^{(8)}$ compatibles con el caso de este paciente. Se conoce que las formas localizadas pueden evolucionar a formas difusas, con afectación de las mucosas hasta en un $6 \%$ de los casos, además, la diseminación leucémica puede ocurrir independientemente de la diseminación cutánea. ${ }^{(4,8,17)}$

En relación al diagnóstico molecular, la positividad expresada por inmunofenotipo para CD4+ y CD56+ ha sido definida por la OMS como el Gold standar para este tipo de leucemia, pero también existe el fenotipo extendido positivo para CD45, HLA-DR, CD123, CD116, BDCA2+, BDCA4 +, con ausencia de CD3, CD11c-, MPO- y CD79a-, cuando el CD56 es negativo el diagnóstico suele ser más desafiante. ${ }^{(2,8,9,16,18)}$ El estudio genético muestra la presencia de los genes TCR e IGH en la configuración de la línea germinal, así como también existen cariotipos complejos y anomalías cromosómicas ${ }^{(8)}$

En las lesiones cutáneas el método diagnóstico es la biopsia, donde se demuestra la presencia de células inmaduras con morfología blástica. (2) Histopatológicamente suele infiltrar la dermis y al tejido subcutáneo, generalmente con mononucleares de mediano tamaño, semejantes a linfoblastos o mieloblastos. ${ }^{(16)}$

Es importante el diagnóstico diferencial con el sarcoma mieloide y el linfoma extranodal de células NK O células T mediante inmunohistoquímica, ${ }^{(5)}$ en algunos casos el diagnóstico sigue siendo un desafío, probablemente debido a la falta de anomalías cromosómicas recurrentes y específicas, sus características superpuestas con otras entidades y su presentación clínica heterogénea con localizaciones tisulares múltiples y variables, en el presente caso la biopsia cutánea demostró únicamente una vasculitis leucocitoclástica no específica.

Un diagnóstico temprano permite un tratamiento óptimo, en casos donde exista afectación únicamente cutánea sin diseminación, se puede emplear radioterapia local, (4) mientras que al tener enfermedad diseminada, existen varios tipos de manejo entre ellos la combinación de quimioterapia a dosis altas como terapia de inducción, basada en CHOP like (ciclofosfamida, doxorrubicina, vincristina y prednisona), DeVIC (carboplatino, etopósido, ifosfamida, y dexametasona), o hyper-CVAD, (8,9) seguida del trasplante alogénico de células hematopoyéticas (TCSH) controla la enfermedad hasta en el 50\% de los casos, con remisión completa de la misma, de acuerdo con los resultados obtenidos por Pagano L, et al. $(4,9)$

La quimioterapia convencional por sí sola no parece ser suficiente para garantizar remisiones duraderas a largo plazo, la tasa de recaída dentro de los 2 años es de aproximadamente el $60 \%$ en pacientes que logran una remisión completa; a pesar de que se ha observado mejores tasas de respuesta con el esquema Hyper-CVAD, que fue el administrado al paciente del caso clínico.

La combinación de ambas terapias incrementa la supervivencia de manera significativa, conforme demostrado en reportes de casos previos, desafortunadamente no existen ensayos controlados aleatorizados por su baja incidencia, por lo que no disponemos de directrices de práctica clínica o lineamientos específicos para el tratamiento. ${ }^{(9,19)}$ 


\section{Conclusión}

El pronóstico de esta enfermedad es sombrío, en virtud de su comportamiento biológico. Su diagnóstico requiere de un equipo multidisciplinar - médicos clínicos y patólogos expertos, para poder proporcionar un tratamiento rápido, teniendo en cuenta el cuadro subclínico que a menudo presenta. El diagnóstico definitivo se basa en el análisis inmunohistoquímico y citofluorimétrico con paneles extendidos, con expresión de CD4+ y CD56 combinado con la expresión de uno o más antígenos específicos de células dendríticas plasmocitoides. No existe evidencia suficiente sobre cuál es el mejor tratamiento, sin embargo, se ha asegurado que la combinación de quimioterapia convencional basada sobre todo en el esquema Hyper-CVAD, junto al TCSH prolonga la sobrevida; tratamiento que no pudo ser completado en este caso por la severa agresividad de la patología.

\section{Referencias}

1. Avilés A, Candelaria M, Ponce M, Cañavera A y Burgos S. Neoplasia blástica de células dendríticas plasmocitoides. Casos clínicos. Rev dríticas plasmocitoides. Casos
Med Chile 2017; 145: 1208-1212

2. Sharma S, Rana C. CD4+ CD56+ Hematodermic Neoplasm Without Cutaneous Involvement. Indian J Hematol Blood Transfus 2014; 30(1):1-3

3. Suzuki Y, Kato S, Kohno K, Satou A, Eladl AE, Asano $N$, et al. Clinicopathological analysis of 46 cases with CD4+ and/or CD56+ immature hematolymphoid malignancy: reappraisal of blastic plasmacytoid dendritic cell and -related neoplasms. Histopathology 2017: $71(6): 972-984$

4. Julia $F$, Petrella $T$, Beylot-Barry $M$, Bagot $M$ Lipsker D, Machet L, et al. Blastic plasmacytoid dendritic cell neoplasm: clinical features in 90 patients. Br J Dermatol 2013;169: 579-586

5. Manoj R, Prabhjot B, Samanjit K, Shilpa K y Rashi S. Blastic plasmacytoid dendritic cell neoplasm. Clinical Case reports 2018; 6(4): 770-772

6. Pemmaraju N. Blastic Plasmacytoid Dendritic Cell Neoplasm. Clin Adv Hematol Oncol 2016 14(4) 220-2

7. Yongxin R, Peihong Z, Shuxu D, et al. Morphologic Characteristics of Blastic Plasmacytoid Dendritic Cell Neoplasm: A Case Report. Ultrastructural Pathology 2014; 38(1): 66-68.
8. Owczarczyk A Soko $\square$ owska M Olszewska B Malek M, Znajewska A, Kowalczyk A, et al, Clinicopathologic retrospective analysis of blastic plasmacytoid dendritic cell neoplasms. Postepy Dermatol Alergol 2018; 35(2): 128-138.

9. Pagano L, Valentini C, Grammatico S y Pulsoni A. Blastic plasmacytoid dendritic cell neoplasm: diagnostic criteria and therapeutical plasm: diagnostic criteria and therapeutical 202

10. Han X, Ouyang $M Q$, Pei Q, et al. Clinical characteristics of 6 patients with blastic plasmacytoid dendritic cell neoplasm. Zhonghua Xue Ye Xue Za Zhi. 2017. 38(8):700-705

11. Kaur V, Swami A, Shebli A et al. A rare case of blastic plasmacytoid dendritic cell neoplasm with deletion 7q.31, in the setting of heavy pre-treatment with alkylating chemotherapy. J Oncol Pharm Pract. 2017. (7):552-556

12. Mohamed A. Kharfan $\square$ Dabaja, Monzr M. A Malki, Uday Deotare, et al. Haematopoietic cell transplantation for blastic plasmacytoid dendritic cell neoplasm: a North American multicentre collaborative study. BJH. 2017. 179(5): 781-789.

13. Bueno C, Almeida J, Lucio P, et al. Incidence and characteristics of CD4+/HLA DRh dendritic cell malignancies. haematologica 2004:89:58-69
14. Petrella $T$, Bagot $M$, Willenze $R$, et al. Blastic NK-Cell Lymphomas (Agranular CD4+CD56+ Hematodermic Neoplasms). Am J Clin Patho 2005:123:662-675 DOI: 10.1309/GJWNPD8HU 5MAJ837

15. Kaabar $M$, Lemaire $P$, Laribi K. Leucémie dérivée des cellules dendritiques plasmocytoïdes: à propos de deux cas. Ann Biol Clin 2015; 73 (6) : $733-6$

16. Zychowska M, Batycka A, Wozniak Z y Maj J. Blastic plasmacytoid dendritic cell neoplasm: a rare lymphoma of extremely aggressive course. Postepy Dermatol Alergol 2017; 34(5): 504-506.

17. Dargent JL, Delannoy A, Pieron P, et al. CU taneous accumulation of plasmacytoid dendritic cells associated with acute myeloid leukemia: a rare condition distinct from blastic plasmacytoid dendritic cell neoplasm. J Cutan Pathol 2011; 38(11):893-8.

18. Galati D, Corazzelli G, De Filippi R. Dendritic cells in hematological malignancies. Crit Rev Oncol Hematol 2016;108:86-96

19. Kerr D y Sokol L. The advances in therapy of blastic plasmacytoid dendritic cell neoplasm. Expert Opin Investig Drugs. 2018 (9):733-739. 